
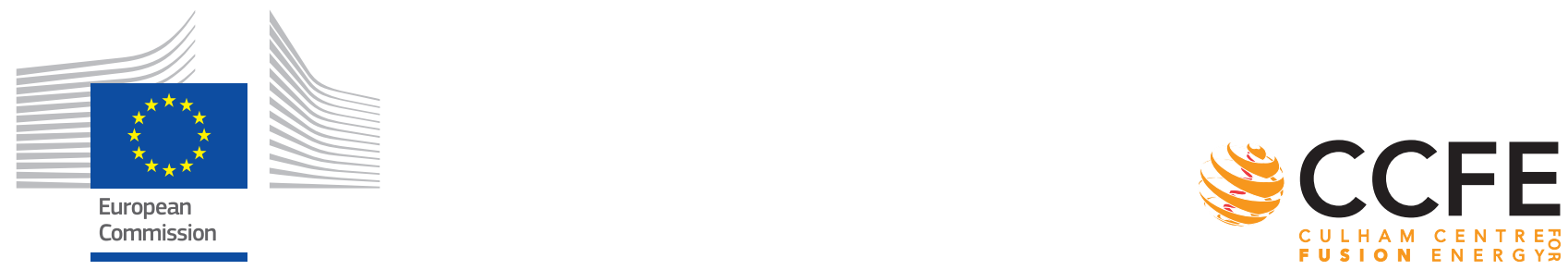

Contract for the Operation of the JET Facilities Co-Funded by Euratom

NJOC-PR(16) 15136

I Balboa et al.

\title{
Recent Developments of In-vessel Calibration of Mid-Infrared Cameras at JET
}

Preprint of Paper to be submitted for publication in 21st Topical Conference on High Temperature Plasma Diagnostics 2016 
This work has been carried out within the framework of the Contract for the Operation

of the JET Facilities and has received funding from the European Unions Horizon 2020 research and innovation programme. The views and opinions expressed herein do not

necessarily reflect those of the European Commission. 
This document is intended for publication in the open literature. It is made available on the clear understanding that it may not be further circulated and extracts or references may not be published prior to publication of the original when applicable, or without the consent of the Publications Officer, EUROfusion Programme Management Unit, Culham Science Centre, Abingdon, Oxon, OX14 3DB, UK or e-mail Publications.Officer@euro-fusion.org

Enquiries about Copyright and reproduction should be addressed to the Publications Officer, EUROfusion Programme Management Unit, Culham Science Centre, Abingdon, Oxon, OX14 3DB, UK or e-mail Publications.Officer@euro-fusion.org

The contents of this preprint and all other EUROfusion Preprints, Reports and Conference Papers are available to view online free at http://www.euro-fusionscipub.org. This site has full search facilities and e-mail alert options. In the JET specific papers the diagrams contained within the PDFs on this site are hyperlinked 


\title{
Recent Developments of In-Vessel Calibration of Mid-IR Cameras at JET ${ }^{\text {a) }}$
}

\author{
I. Balboa,,${ }^{1, b)}$ S. Silburn, ${ }^{1}$ P. Drewelow, ${ }^{2}$ V. Huber,${ }^{3}$ A. Huber, ${ }^{4}$ D. Kinna, ${ }^{1}$ M. Price,,${ }^{1}$ \\ G. F. Matthews, ${ }^{1}$ S. Collins, ${ }^{1}$ J. Fessey,${ }^{1}$ M. Rack, ${ }^{4}$ P. Trimble, ${ }^{1}$ and K-D. Zastrow ${ }^{1}$ \\ ${ }^{1}$ CCFE Fusion Assoc., Culham Science Centre, Abingdon, OX14 3DB, UK \\ ${ }^{2}$ Max-Planck Institute für Plasma Physik, EURATOM Association, 17491 Greifswald, Germany \\ ${ }^{3}$ Jülich Supercomputing Centre, Research Centre Jülich, 52425 Jülich, Germany \\ ${ }^{4}$ Forschungszentrum Jülich GmbH, Institut für Energie-und Klimaforschung - Plasmaphysik, Partner of the \\ Trilateral Euregio Cluster (TEC), 52425 Jülich, Germany
}

(Presented XXXXX; received XXXXX; accepted XXXXX; published online XXXXX)

(Dates appearing here are provided by the Editorial Office)

Recent improvements in software tools and methodology have allowed us to perform a more comprehensive in-vessel calibration for all mid-infrared camera systems at JET. A comparison of experimental methods to calculate the NUC is described as well as the linearity for the different camera systems. Measurements of the temperature are assessed for the different diagnostics.

\section{INTRODUCTION}

JET infrared scientific cameras operate in the middle wavelength infrared range (MWIR: 3-5 $\mu \mathrm{m}$ ). They can produce a map of the temperature and power deposition to the plasma facing components which are included within their field of view. The basic principle is that the infrared (IR) radiation emitted by the component's surface can be imaged onto the camera. The raw signal is commonly known as "Digital Levels" (DL). Once the calibration is applied and together with the emissivity of the target, it is then possible to produce a temperature measurement as a function of time. There are a total of 4 scientific MWIR CMOS cameras. Two systems (KL9A-E5TA and KL9B-E8TA) view the bulk tungsten made central tile of the divertor through a vertical port, on top of the machine. These systems are located at different octants which allows the study of toroidal asymmetries. The other two (KL7-E8WB and KL3B-E4DA) look toroidally through complex endoscopes. KL7-E8WB provides a wide angle view and KL3B-E4DA provides a split image of two separate views of vertical divertor tiles for two different locations. An overview of the performance of these diagnostics can be found in a previous publication ${ }^{1}$. In the past, due to resource and time constraints, it has only been possible to carry out an independent calibration for KL7-E8WB, which meant that for the other diagnostics a relative calibration had to be applied. Recent improvements in the software and methodology have allowed us to perform an in-vessel calibration for all four diagnostics with an improved level of traceability.

\section{IN-VESSEL CALIBRATION}

For the in-vessel calibration, a grey body is brought inside the vessel and placed in front of the field of view of the camera system by a robotic arm. The grey body that was used had a heating element of dimensions $200 \mathrm{~mm} x 200 \mathrm{~mm}$. The temperature ranges goes between $200-600^{\circ} \mathrm{C}$. The emissivity has been

${ }^{a}$ Contributed paper published as part of the Proceedings of the 21st Topical Conference on High-Temperature Plasma Diagnostics (HTPD 2016) in Madison, Wisconsin, USA.

b) Author to whom correspondence should be addressed:

itziar.balboa@ukaea.uk. measured to be $\sim 0.7$ for a wavelength of $4 \mu \mathrm{m}$. The grey body was built in-house, it consists of 12 cartridge heaters equally spaced across a stainless steel plate which is coated with SiC. Six thermocouples are embedded across the heating plate. The plate is integrated inside an aluminium alloy box and insulated with a high temperature refractory board. The housing box has been designed to be handled by the robotic arm. The source is fed by a $110 \mathrm{~V}$ power supply and an Ethernet cables that allows the control of the source remotely (total heating power $<2 \mathrm{~kW}$ ). The source takes a minimum of 30 minutes to reach $600^{\circ} \mathrm{C}$ and $\sim 8 \mathrm{~h}$ to cool down to room temperature.

A package of software tools was developed internally to carry out the acquisition, triggering and recording to produce all the data. Additional tools ("JUVIL") have been developed to watch video images, select a region of interest (ROI), averaging frames and calculate individual profiles as a function of time. These are being described in another article at this conference ${ }^{2}$. An editor enables the users to fill in the relevant parameters. The acquisition starts when the camera receives a "virtual" software trigger that sets the start of the recording. Each measurement consists of two files. First, the "header" file that is a "json" file that contains all the information about the set up (i.e. camera, optics, filter, hot source...) and the second file is the recording video. Each measurement is uniquely named by using a Unix timestamp. The measurements are then pushed to the central server where it is safely stored. Furthermore, a different set of programs was then later written to produce the calibration curves. These programs are available in the CCFE GitLab repository.

\section{NON-UNIFORMITY CORRECTION}

The response of the individual camera pixels is not uniform across the sensor. This non-uniformity leads to the creation of artefacts onto the image that result eventually in measurement inaccuracies. Therefore, it is necessary to implement a "nonuniformity correction" (also known as "NUC") to all pixels in the sensor. There are two key parameters: pixel gain and offset. Mathematically, the raw digital signal, DL, can be expressed $a^{3}$ : 


$$
\mathrm{DL}=\mathrm{C}+\mathrm{t}_{\mathrm{exp}} \cdot \mathrm{O}+\mathrm{t}_{\exp } \cdot \mathrm{G} \cdot \mathrm{L}(\mathrm{T}, \tau, \xi)
$$

where "C" represents a constant associated to the electronics offset due to the conversion of the electrical signal to digital one; "texp" denotes exposure time, "O" is the pixel offset which includes effects such as dark current, etc.., "G" specifies the pixel gain and " $\mathrm{L}$ " means the radiation coming from the target as well as the optical path and its components. The offsets " $\mathrm{C}$ " and "O" can be measured by placing a gold mirror in front of the lens and taking images of the sensor for different exposures. Here it is assumed that the radiance reflected by the mirror is negligible since the sensor temperature is $77 \mathrm{~K}$. The gain is obtained by recording images from a cold and hot source placed in front of the camera lens. Mathematically solving a system of two linear equations following the equation given in [1] for two different temperatures and the same exposure time, allows you to derive the offsets and the relative gain for each pixel with respect to a reference pixel (usually the central pixel). Applying the NUC means scaling the gain and the offset of all pixels relative to the reference pixels. The key properties required for any radiation source used for NUC measurements is uniformity and stability. To this effect, three radiation sources were compared: water in a glass jar, water in a plastic container and a grey body source described in section II. The equipment was set up in the lab since it is possible to position the camera close to the source. The NUCs obtained using the water type sources and the grey bodies were applied onto a few raw images. The best result (i.e. most uniform) appears to be the NUC corresponding to the grey body. The water based sources are not as good but the plastic container filled with water produces a reasonable result. The reason being that because the temperature difference between the cold and hot frames for the water type source is only a few tens of degrees. Thus, it is more challenging to identify all the "bad" pixels. A pixel is considered "bad" when its intensity varies by more than 3 standard deviations from its smooth neighbours.

The radiance of the grey body source can vary across the surface and therefore it is important to look at larger areas to get the most stable result. Once the grey body was selected as the preferred source, NUCs were obtained for different integration times. A linear fitting combining of all the NUCs allows the interpolation of the gain and the offset for any particular integration time. In addition, in three cameras a correlation was found between the position of "bad" pixels in the frame and whether the frame number was odd or even, (e.g. a pixel is "bad" only in odd frames). A possible explanation for this phenomenon could be related to the integrator capacitors used during the integration of the signal. Figure 1 shows how a pixel "A" has a higher intensity for all the even frames than the odd frames and vice versa for pixel "B". Consequently, a pair of NUCs, each one associated with a specific parity (even/odd), is now currently produced for every pulse. The name given to these NUCs is "Flip-NUC". Furthermore, it is also necessary to identify which Flip-NUC is applied to which frame parity since the camera is running in free mode, therefore the parity of odd/even is just relative. The combination that gives the minimum difference is the one that should be used for the entire pulse. The improvement associated with the Flip-NUCs has been calculated by comparing the standard deviation across an average of odd frames (or even frames) for the case when: a standard NUC is applied to correct the image versus a Flip-NUC is applied to the same image. The ratio between the two standard deviations was found to vary between 1.1 to a factor 3 improvement. This was performed for a range of integration times.
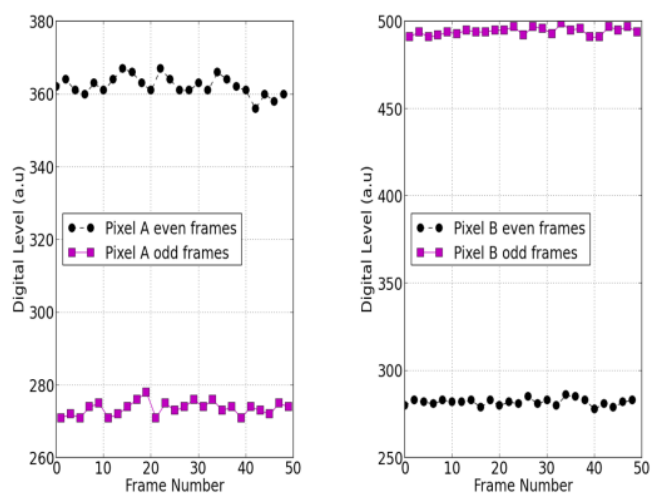

Figure 1. Distribution of bad pixels for odd and even frames as function of the frame number

\section{LINEARITY RESPONSE}

Comparing the linear sensitivity of the different diagnostics showed that for the wide angle view camera diagnostic, the relation between the raw signal and the integration time was not linear, in particular for relatively low signal levels (less than 25\% of the maximum digital level). This jeopardizes not only the implementation of the NUC but also the calibration which assumes a linearity between digital counts and photon flux. Consequently, a look up table was introduced to translate the non-linear range of the signal into a linear one before applying the NUC and the calibration.

\section{TEMPERATURE MEASUREMENT}

One of the key advantages of the in-vessel calibration is that it takes into account the current performance of the optical components included in the optical path. Therefore, the calibration does not rely on any assumptions or specification values from a data sheet but on actual performance. In addition, it would not be feasible to carry out a calibration with the entire diagnostic in the lab. The only MWIR diagnostic where the old and the new calibration have been in-vessel is the wide angle view KL7-E8WB. Figure 2 shows a comparison of the old and new calibration curves for Beryllium since this is the material of the inner and outer wall limiters that cover most of the field of view for this diagnostic. The temperature difference at $1400^{\circ} \mathrm{C}$ between the two curves is slightly higher than $250^{\circ} \mathrm{C}$. The discrepancy between the two curves is attributed first to the implementation of the non-linearity correction in the raw data which was identified during the last in-vessel calibration. Secondly, the emissivity value used for bulk Beryllium has also been changed ${ }^{4}$. For example at $4 \mu \mathrm{m}$ and $900^{\circ} \mathrm{C}$, the new value is $0.30 \pm 0.03$ in comparison with 0.22 used before for all wavelengths. Inside the graph, there is a second plot which shows the in-vessel data and the calibration fit for 3 different filters

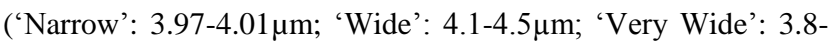
$4.6 \mu \mathrm{m})$. There is good agreement between in-vessel data and calibration fit. The uncertainty of the in-vessel measurements is \pm $1 \sigma$.

For the KL9A-E5TA and KL9B-E8TA (both looking to the divertor), the camera units were swapped and the emissivity values for bulk and coated tungsten updated ${ }^{4}$. As an example, for $4 \mu \mathrm{m}$, the emissivity for bulk tungsten at $900^{\circ} \mathrm{C}$ is $0.12 \pm 0.01$ instead of 0.084 for the previous calibration. For KL9A-E5TA an optical filter was added to the optical path. Thus, a significant change in the old (i.e. indirect method) and new (i.e. in-vessel) 
calibration vectors is expected for KL9A-E5TA (see figure 3, left hand side). However, for KL9B-E8TA, the swapping of the cameras and the change in emissivity do not account for a difference in digital levels of a factor slightly higher than 1.5 between the calibration curves at $3500^{\circ} \mathrm{C}$ (see figure 3 , right hand side).

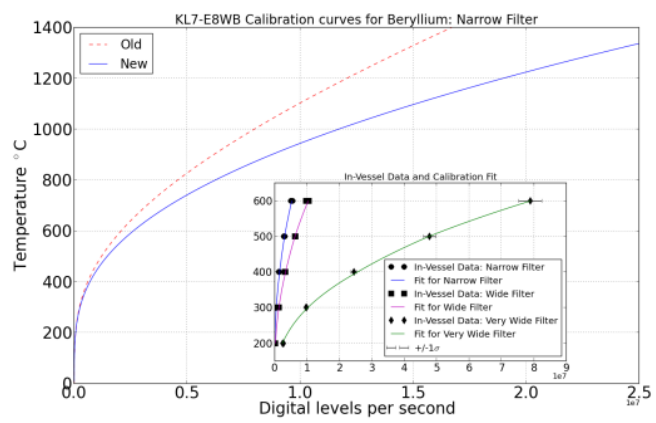

Figure 2. Comparison of calibration curves for old/new in-vessel calibrations for KL7-E8WB. Inside the graph is shown the invessel experimental data and the calibration fit for narrow, wide and very wide filters.

It has not been possible to identify a reason for the change, however when comparing identical JET pulses (89060 and 85414 ) between the previous and the last campaign, the raw signal in the two pulses shows the same ratio as the calibration curves. Therefore, both calibrations are considered credible.
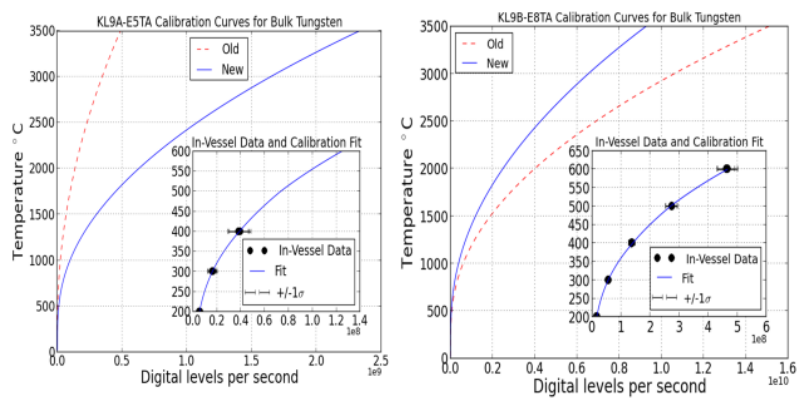

Figure 3. Comparison of the old (i.e. indirect method) and new (i.e. in-vessel) calibration curves for bulk tungsten for KL9AE5TA (left) and KL9B-E8TA (right). Inside the graph is shown the in-vessel experimental data and the fitted calibration curve respectively.

Finally, comparing the old (i.e. indirect) and new (i.e. in-vessel) calibration curves for KL3B-E4DA, the difference in digital levels is slightly higher than a factor of 2 (see figure 4) at $3500^{\circ} \mathrm{C}$. The key changes are the implementation of the NUC and the new emissivity values for bulk tungsten and for tungsten coated tiles $^{4}$ (not shown in figure 4). However these changes do not justify the large difference in the two calibration results. Since it is not possible to measure the transmission of the endoscope independently, it can only be assumed that either this has changed or the previous cross-calibration was not sufficiently accurate. Figure 5 compares the peak temperatures for one pulse, 88733, for the divertor IR cameras. KL9A-E5TA and KL9BE8TA are within $\pm 50^{\circ} \mathrm{C}$ for stack $\mathrm{C}$ of tile \#5. This pulse had two heating phases. On the other hand KL3B-E4DA shows a similar profile but the temperature is around $250^{\circ} \mathrm{C}$ lower. This can be explained due to the difference in the spatial resolution between KL3B-E4DA and the other two camera systems.

The emissivity for Beryllium, bulk Tungsten and Tungsten coated has been measured experimentally ${ }^{4}$ and extrapolated to other temperatures ${ }^{5}$. Regarding the impact of reflections, the wide angle KL7-E8WB is more strongly affected than the other divertor systems.

The effect shows as an increase of the temperature of the inner limiter when the divertor appears to be heating up significantly.

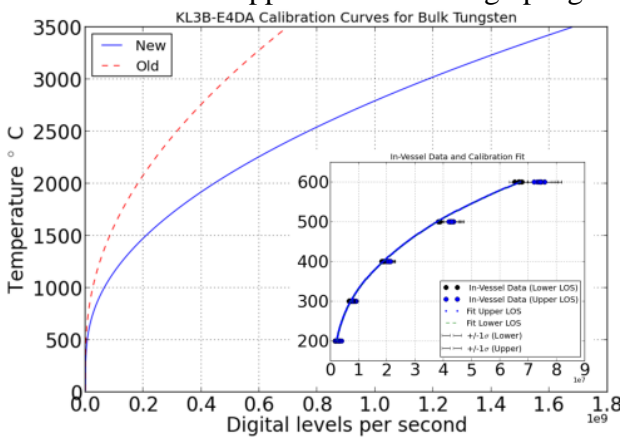

Figure 4. Comparison of the old (i.e. indirect) and new (i.e. invessel) calibrations for bulk tungsten for KL3B-E4DA. Inside the graph is shown the in-vessel experimental data and the fitted calibration curve respectively.

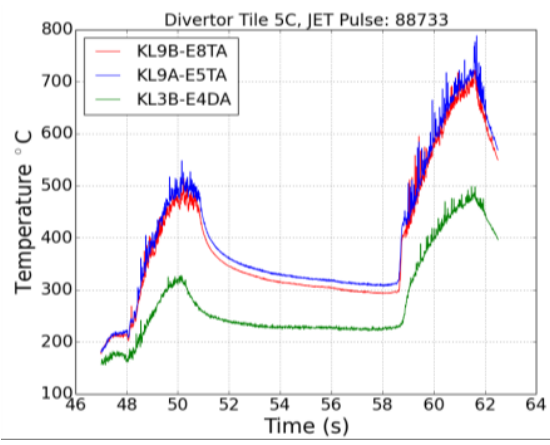

Figure 5. Comparison of divertor tile \#5 (stack C) temperatures for the different divertor IR cameras for pulse JET 88733.

\section{CONCLUSIONS}

The in-vessel calibration of all MWIR camera diagnostics has provided a direct method to characterize these diagnostics. Significant differences have been noted from the previous indirect method. The in-vessel approach relies on the uniformity and stability of the grey body source. Implementation of a NUC based on the parity of the frame improves the correction of artifacts in the image.

\section{ACKNOWLEDGMENTS}

This work has been carried out within the framework of the Contract for the Operation of the JET Facilities and has received funding from the European Union's Horizon 2020 research and innovation programme. The views and opinions expressed herein do not necessarily reflect those of the European Commission.

\section{REFERENCES}

${ }^{1}$ I. Balboa et al., Rev. Sci. Instrum.83, 10D530 (2012)

${ }^{2} \mathrm{~V}$. Huber et al., these proceedings

${ }^{3} \mathrm{http}: / / d x . d o i . o r g / 10.14279 /$ depositonce-3823

${ }^{4}$ C. Russet et al., WPJET1 report-RO. Thermal emissivity at $4 \mu \mathrm{m}$ for W coated CFC tiles determined with a Single Pixel Scanning IR Camera (to be published)

${ }^{5}$ CRC Handbook of Chemistry and Physics, $75^{\text {th }}$ ed., edited by D. R. Lide and H. P. R Frederick (CRC, 1994) 